\title{
Performance Comparison of Ad hoc Routing Protocols under High Mobility Environment
}

\author{
Supriya Gupta \\ Dept. of Computer Sc. \& IT \\ University of Jammu \\ Jammu, India
}

\author{
Dr. Lalitsen Sharma \\ Dept. of Computer Sc. \& IT \\ University of Jammu \\ Jammu, India
}

\begin{abstract}
Routing in mobile ad hoc network is considered a challenging task due to the unpredictable changes in the network topology, resulting from the random and frequent movement of the nodes and due to the absence of any centralized control. Several routing protocols for mobile ad-hoc networks are being proposed. In this paper the performance of two major routing protocols for mobile ad hoc networks: Dynamic Source Routing (DSR) and Destination Sequence Distance Vector routing (DSDV), is measured under high mobility environment using NS-2 simulator. The performance is analyzed on basis of average endto-end delay, throughput and jitter.
\end{abstract}

\section{General Terms}

Performance Comparison

\section{Keywords}

mobile; ad hoc; DSR; DSDV; simulator; throughput.

\section{INTRODUCTION}

The mobile ad hoc networks are becoming increasingly popular due their less cost and rapid deployability; inherent support for mobility and the potential to provide ad hoc connectivity to devices, to form temporary networks in areas where there is no fixed infrastructure. In such a network, each mobile node operates as a host as well as a router, forwarding packets for other mobile nodes in the network, that are not in direct wireless transmission range of each other [1]. Development of efficient routing protocols for mobile ad hoc networks is a challenging task as due to the node movements, the network topology can change randomly and rapidly at unpredicted times and also the bandwidth is limited and can vary due to fading, noise and interference. Many different protocols have been proposed to solve the routing problem in ad hoc networks and are broadly classified as proactive and reactive routing protocols [2]. In Proactive routing protocols like DSDV [3], CGSR [4], OLSR [5], GSR [6] , the nodes maintain consistent and up-to date routing information from each node to all other nodes by exchanging routing information periodically. The main advantage of proactive routing is when a source needs to send packets to a destination, the route is already available, i.e., there is no latency and the disadvantage is that some routes may never be used and broadcasting of routing information will consume a lot of the scarce resources like bandwidth, buffers etc. In case of reactive routing protocols such as DSR [7], AODV [8], SSA [9], the routing information is updated only when a routing requirement is presented, saving the network bandwidth. In this paper the two protocols DSDV, a proactive routing protocol, and DSR, a reactive routing protocol, are briefly reviewed and a simulation based comparative performance analysis is presented. Our results are based on simulations of an ad hoc network of 50 wireless mobile nodes randomly moving about and communicating with each other. The Protocols are compared on the basis of the performance metrics viz throughput, average end-to-end delay and jitter. The following section gives short descriptions of the two ad-hoc routing protocols studied in this paper.

\section{PROTOCOL DESCRIPTIONS}

\subsection{Destination Sequenced Distance Vector - DSDV}

DSDV [3] is a hop-by-hop distance vector proactive routing protocol in which each network node maintains a routing table that contains the next-hop for and number of hops to all reachable destinations. Periodical broadcasts of routing updates keep the routing table completely updated at all times. To guarantee loop-freedom, DSDV uses a concept of sequence numbers that indicate the freshness of a route. A route $\mathrm{R}$ is considered more favorable than $\mathrm{R}^{\prime}$ if $\mathrm{R}$ has a greater sequence number or, if the routes have the same sequence number but $R$ has a lower hop-count. The sequence number for a route is set by the destination node and increased by one for every new originating route advertisement. The stations periodically transmit their routing tables to their immediate neighbors as well as when a significant change has occurred in its table from the last update sent. So, the update is both time-driven and eventdriven. The parameter values used for DSDV in the simulation are:

- Periodic route update interval $-15 \mathrm{~s}$

- Periodic updates missed before link declared broken 3

- Route advertisement aggregation time -1s

- Maximum packets buffered per node per Destination $-5$

\subsection{Dynamic Source Routing - DSR}

Dynamic Source Routing [7] is a reactive routing protocol which uses source routing to deliver data packets. Headers of data packets carry the sequence of nodes through which the packet 
must pass. This means that intermediate nodes only need to keep track of their immediate neighbors in order to forward data packets. The source, on the other hand, needs to know the complete hop sequence to the destination. The route acquisition procedure in DSR requests a route by flooding a Route Request packet. A node receiving a Route Request packet searches its route cache, where all its known routes are stored, for a route to the requested destination. If no route is found, it forwards the Route Request packet further on after having added its own address to the hop sequence stored in the Route Request packet. The Route Request packet propagates through the network until it reaches either the destination or a node with a route to the destination. If a route is found, a Route Reply packet containing the proper hop sequence for reaching the destination is unicasted back to the source node. To avoid unnecessarily flooding the network with Route Request messages, the route acquisition procedure first queries the neighboring nodes to see if a route is available in the immediate neighborhood by sending a first Route Request message with the hop limit set to zero, thus it will not be forwarded by the neighbors. If no response is obtained by this initial request, a new Route Request message is flooded over the entire network. The parameter values used in the DSR simulations are:

- Time between retransmitted requests- $500 \mathrm{~ms}$

- Size of source route header carrying $\mathrm{n}$ addresses bytes $4 n+4$

- Time-out for non propagating search- $30 \mathrm{~ms}$

- Time to hold packets awaiting routes-30s

- Maximum rate for sending replies for a route- $1 / \mathrm{s}$

\section{SIMULATION MODEL}

\subsection{Network Simulator}

The entire simulations were carried out using ns2.34 network simulator which is a discrete event driven simulator developed at University of California Berkeley [10] as a part of the VINT project [11]. It provides substantial support for simulation of TCP, routing, multicast protocols over wired and wireless (local and satellite) networks, etc. The simulator is event-driven and runs in a non-real-time fashion. It consists of $\mathrm{C}++$ core methods and uses $\mathrm{Tcl}$ and Object Tcl shell as interface allowing the input file (simulation script) to describe the model to simulate. Arbitrary network topologies composed of nodes, routers, links can be defined. A rich set of protocol objects can be attached to nodes, usually as agents. It has become the de facto standard in networking research [12].

\subsection{Traffic Generation Models}

Traffic-scenario generator script 'cbrgen.tcl' is used to create constant bit rate (CBR) traffic connections between wireless mobile nodes. . The maximum number of connections setup between the nodes was 20 , traffic rate of 5 packets per seconds where each packet size was 512 bytes. A set of two traffic generation files corresponding to each routing protocols were used for same values of maximum connections.

\subsection{Mobility Generation Models}

For nodes positions and their movement, a mobility generation file is created with the statements which set nodes' positions and nodes movement using CMUs scenario generation tool setdest [12]. These files are characterized by a pause time. To study the effect of high node mobility, the simulation is carried out with movement patterns generated for a pause time of 0 seconds using the 'random waypoint algorithm'. The simulation parameters used are:

- Number of nodes- 50

- Simulation Time- 100sec.

- Area $-500 \mathrm{~m} * 500 \mathrm{~m}$

- Max Speed $-20 \mathrm{~m} / \mathrm{s}$

- Traffic Source- TCP

- Pause Time (sec)- $0 \mathrm{sec}$

- Packet Size- 512 Bytes

- Packets Rate- 4 Packets/s

- Max Connections-20

- Band Width- 10Mbps

- Mobility model used-- Random way point

The routing protocols are evaluated at pause time set to 0 s, where pause time is the stop time between successive movements of nodes and $0 \mathrm{~s}$ denotes high mobility.

\section{METRICS USED FOR ANALYSIS}

The following metrics were used for the comparison of the protocols:

\subsection{End to End delays:}

It represents the delay encountered between the sending and receiving of the packets.

\subsection{Throughput of received packets:}

Throughput is the measure of how fast we can actually send through network. This represents the number of packets received within a given time interval.

\subsection{Jitter:}

It represents any unwanted variation in delays generated during the packet transfer.

\section{RESULTS AND ANALYSIS}

The following table (Table 1) summarizes the result of simulation parameters to evaluate the performance of the two routing protocols. 
Table 1. Resultant Simulation Parameters

\begin{tabular}{|c|c|c|}
\hline Parameters & DSR & DSDV \\
\hline $\begin{array}{c}\text { Number of } \\
\text { generated packets }\end{array}$ & 15599 & 14308 \\
\hline $\begin{array}{c}\text { Number of sent } \\
\text { packets }\end{array}$ & 15506 & 14220 \\
\hline $\begin{array}{c}\text { Number of } \\
\text { forwarded packets }\end{array}$ & 1897 & 92 \\
\hline $\begin{array}{c}\text { Number of dropped } \\
\text { packets }\end{array}$ & 159 & 0.001937026 \\
\hline $\begin{array}{c}\text { Number of lost } \\
\text { packets }\end{array}$ & 0.001936380 & 72.69449107 \\
\hline $\begin{array}{c}\text { Minimal delay } \\
\text { (sec) }\end{array}$ & 36.86088256 & 0.3557508646 \\
\hline $\begin{array}{c}\text { Maximal delay } \\
\text { (sec) }\end{array}$ & 0.4021218357 & 9 \\
\hline Average delay(sec) & & \\
\hline
\end{tabular}

\subsection{End-to-End Delay}

Average end-to-end delay includes all possible delays caused by buffering during route discovery, latency, queuing at the interface queue, retransmission delays at the MAC, and propagation and transfer times of data packets. Figure 1 shows the end to end delay frequency distribution of the simulated network. DSDV showed less delay in comparison to DSR. However, DSR has a lower delay than DSDV at higher mobility values as the route acquisition procedure in DSR allows more routes to be detected and cached than in DSDV, which obtains a single route per RREQ. Also with DSR, packets wait for lesser time during route acquisition. But DSDV exhibits a low delay because only packets belonging to valid routes at the sending instant get through. A lot of packets are lost until new (valid) route table entries have been propagated through the network by the route update messages in DSDV. For DSR, on the other hand, the reactive route acquisition procedures manage to provide new routes with a low packet loss. Figure 2 shows the cumulative sum of numbers of all the packets dropped during transmission via DSR and DSDV ad hoc routing protocols respectively.

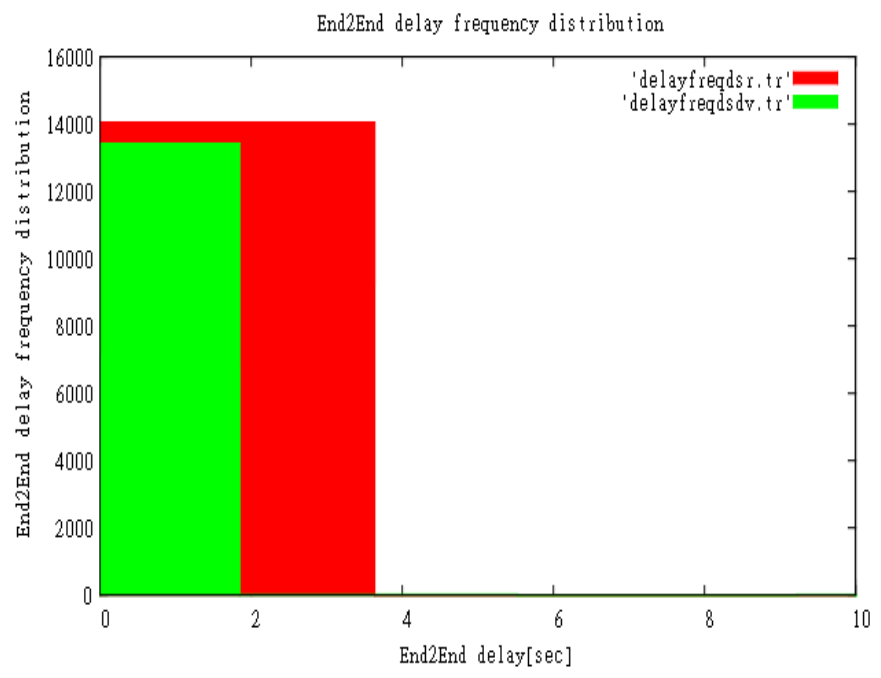

Figure 1. End2End delay frequency distribution

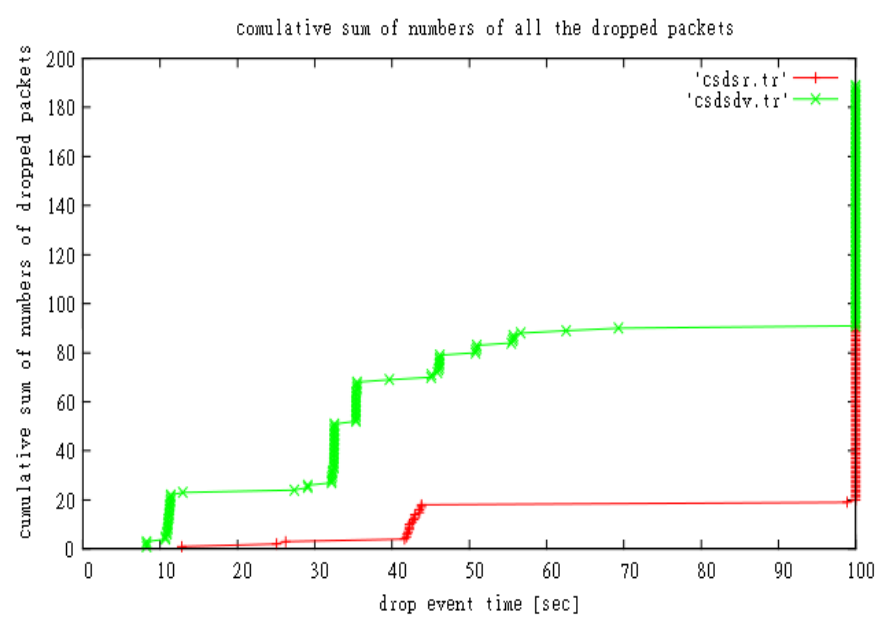

Figure 2. Comulative sum of number of all dropped packets

\subsection{Throughput}

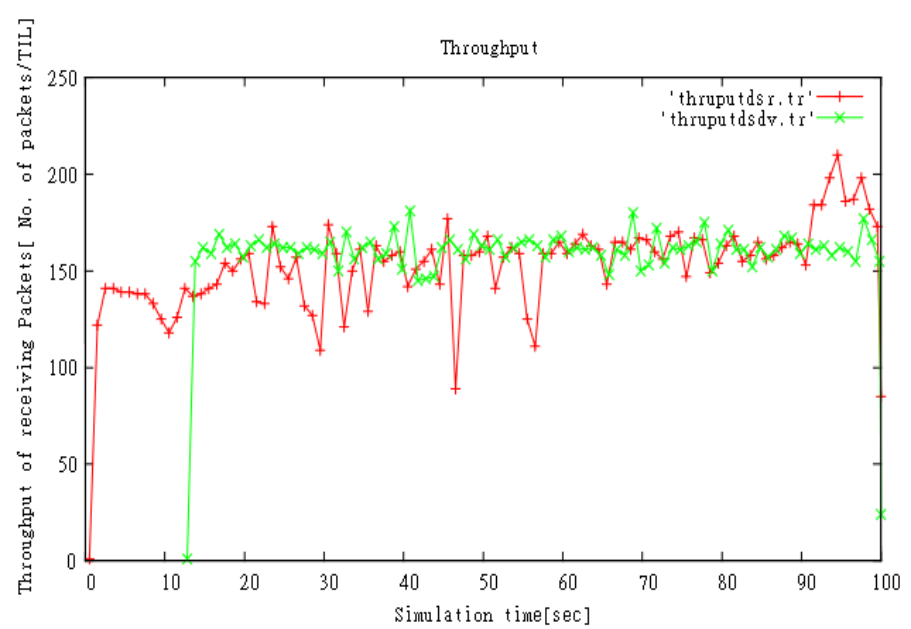

Figure 3. Throughput. (TIL- Time interval Length)

Figure 3 shows the throughput of DSDV and DSR protocols for the simulated network. According to the graph the DSR protocol performs better than DSDV. This is due to fact that at high mobility there will be frequent and high degree of changes in the network topology.

The proactive nature of DSDV makes it less adaptive to this frequent change and greater number of full dumps needs to be exchanged between the nodes in order to maintain up-to-date routing information at the nodes. This huge volume of control traffic consumes a significant part of the channel bandwidth and lesser channel capacity is left for the data traffic which results in reduced throughput fraction of DSDV at higher mobility. Moreover, in DSDV packets are dropped due to stale routing table entry. DSDV keeps track of only one route per destination. Due to lack of alternate routes, MAC layer drops packets that it is unable to deliver through stale routes. DSR on the contrary, is more adaptive to the frequently changing scenario due to its ondemand routing nature.

In case of DSR, multiple routes exist in the cache. Thus, even if a link is broken due to high mobility, alternative routes can be 
found from the cache. The lower throughput for DSDV during initial time interval (Figure 4) is caused by packets that are sent before routes have converged initially in the network. Note that all simulations are started without any established routes.

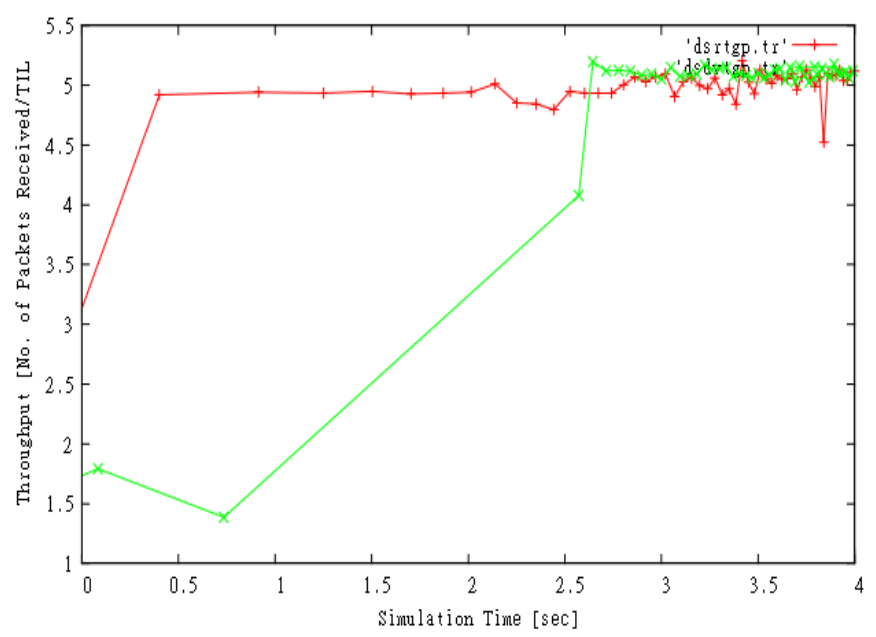

Figure 4. Throughput

\subsection{Jitter}

The Jitter experienced by these protocols cannot to be quantified and they can only be bounded within a delay range. The jitter experienced by DSDV (Figure 5) is the highest than the jitter experienced by DSR

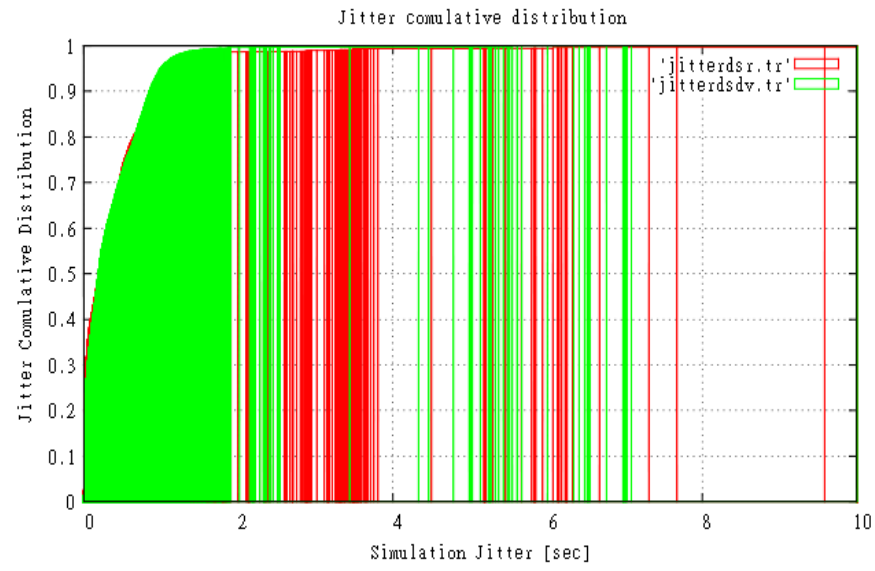

Figure 5. Jitter Comulative Distribution

\section{CONCLUSION}

The protocols under study, namely, DSDV and DSR, cover a range of design choices, including periodic advertisements vs. on demand route discovery, hop-by hop routing vs. source routing. This study clearly indicates that in a network of highly dynamic topology, a reactive routing protocol is superior to a proactive one. DSDV have considerable difficulties in maintaining valid routes and drops many packets. With increasing mobility, its' strive to continuously maintain routes to every node increases network load as updates become larger. DSR, on the other hand, is efficient in finding routes in terms of the number of control mobility; alternative routes can be found from the cache. This prevents packet dropping and results in better packet delivery performance and throughput of DSR. Thus we can conclude that if routing delay is of little concern, then DSR shows better performance than DSDV at higher mobility.

\section{ACKNOWLEDGMENTS}

The authors are thankful to University Grants Commission (UGC), and Ministry of Human Resource Development (MHRD), Government of India for providing financial assistance to carry out this research work. The authors are also thankful to Prof. Devanand, Head, Department of Computer Science and IT, University of Jammu, for his kind support.

\section{REFERENCES}

[1] M. Scott, P. Joseph, H. Gregory 1999. Internet based mobile sd hoc networking. IEEE Internet Computing Magazine.

[2] R. Rajaraman 2002.Topology control and routing in ad hoc networks: a survey. ACM SIGACT News, vol. 33(2), pp.60-73.

[3] Charles E. Perkins and Pravin Bhagwat 1994. Highly dynamic Destination-Sequenced Distance-Vector routing (DSDV) for mobile computers. In Proceedings of the SIGCOMM '94 Conference on Communications Architectures, Protocols and Applications, pages 234-244.

[4] W. Liu, C. Chiang, H. Wiu \& C. Gerla 1997. Routing in clustered multihop mobile adhoc networks with fading channel. In Proc. IEEE SICON'97, pp. 197-211.

[5] T. Clausen \& P. Jacquet 2003. Optimized Link State Routing Protocol (OLSR). rfc-3626.

[6] Tsu-Wei Chen \& Mario Gerla 1997. Global State Routing: A New Routing Scheme for Ad-hoc Wireless Networks. In Proc. IEEE SICON'97, pp. 197-211.

[7] D. Johnson, Y.hu \& D. Maltz 2007. The Dynamic Source Routing Protocol (DSR) for Mobile Ad Hoc Networks for IPv4. rfc-4728.

[8] C. Perkins, E. Belding-Royer \& S. Das 2003. Ad hoc OnDemand Distance Vector (AODV) Routing", rfc-3561.

[9] R.Dubey, D. Rais, K. Y. Wang \& S.K. Tripathi 1996. Signal stability based adaptive routing (SSA) for ad-hoc mobile networks. Computer Science Technical Report Series; Vol. CS-TR-3646, pp. 22.

[10] Network Simulator- ns-2. http://www.isi.edu/nsnam/ns.

[11] The CMU Monarch Project: The CMU Monarch Projects Wireless and Mobility Extensions to ns URL: http://www.monarch.cs.cmu.edu (1998).

[12] Yinfei Pan, "Design Routing Protocol Performance Comparision in NS2: AODV Comparing to DSR as Example", Deptt of CS, SUNY Binghamton, Vestal NY 13850.

[13] J. Broch, D. Maltz, D. Johnson, Yih-Chun Hu \& J. Jetcheva 1998. A performance Comparison of Multi-hop Wireless Ad Hoc Network Routing Protocols . Mobicom'98, Dallas Texas, pp.25-30.

[14] J.Geetha \& G. Gopinath 2007. Ad-hoc Mobile Wireless Networks Routing Protocols. A Review, Journal of Computer Science, pp. 574-582.

[15] N.S. Yadav \& R.P.Yadav 2007. Performance Comparison and Analysis of Table- Driven and On- Demand Routing Protocols for Mobile Adhoc Networks. International Journal of Information Technology, Vol.4, No.2,pp101-109. 